\title{
CATÁLOGO ILUSTRADO DE LOS ESPECÍMENES TIPO DE TIBURONES CUBANOS
}

\author{
Elena Gutiérrez de los Reyes, María V. Orozco, Rolando Cortés, Alina Blanco, \\ Yusimi Alfonso, Daniel Delgado, Elida Lemus e Isis L. Álvarez \\ Departamento de Biodiversidad Marina,Acuario Nacional de Cuba, \\ Ave. Primera y Calle 60, Miramar, Playa, La Habana, Cuba. \\ elenag@acuarionacional.cu
}

\section{RESUMEN}

Se recopila información de diferentes bases de datos para presentar los especímenes tipo de 19 especies de tiburones cubanos (Clase CHONDRICHTHYES), con ilustraciones y datos asociados. Siete de las especies fueron descritas por Don Felipe Poey y Aloy, conocido naturalista cubano del siglo XIX. Los resultados se han ordenado a modo de catálogo ilustrado, con la sistemática actualizada, la institución que las atesora y su número catalográfico, la localidad tipo, la referencia y el título del trabajo correspondiente. Para algunas especies se ofrece la fecha de colecta, así como la profundidad y la expedición en que se colectaron los ejemplares; en ocasiones también aparece el colector. La información ofrecida ha estado dispersa hasta el momento, por lo que esta publicación constituye una recopilación de mucho valor y utilidad.

Palabras clave: catálogo ilustrado, especímenes tipo, tiburones cubanos.

Title: Ilustrated catalog of type specimens of cuban sharks.

\section{ABSTRACT}

The information from different databases is collected to present the type specimens of 19 Cuban sharks (Class CHONDRICHTHYES) with their originals illustrations and associated data. Seven of them are Poey's specimens, a famous Cuban naturalist from 19th century. The results are presented as an illustrated catalog, with updated systematics, the depositary institution and catalog number in the collection, type locality, bibliographic reference and the title of the paper. For some species also include the collection date, the expedition name and sea depth; occasionally the name of collector as well. All this data was dispersed until now, so it is valuable and useful information.

Keywords: illustrated catalog, type specimens, cuban sharks.

\section{INTRODUCCIÓN}

La mayor parte de los especímenes tipo de las especies cubanas, de todos los grupos zoológicos y botánicos marinos conocidos, se encuentran depositados en colecciones de varias instituciones del mundo y su información en bases de datos no siempre se encuentra disponible en línea para ser consultada; lo mismo ocurre con la literatura donde aparecen sus descripciones, son disímiles revistas, no todas digitalizadas y a disposición en la red mundial.

Las expediciones de colecta realizadas alrededor de Cuba han sido numerosas a través de los años, sobre todo por parte de los científicos norteamericanos, quienes han descrito una buena parte de nuestra ictiofauna, razón por la cual los especímenes tipo fueron depositados en instituciones de Estados Unidos de Norteamérica, principalmente en Museum of Comparative 
Zoology (Harvard University) y National Museum of Natural History (Smithsonian Institution). También, la mayoría de los 106 especímenes tipo de especies descritas por Don Felipe Poey fueron enviados por él mismo a dichas instituciones, a través de su colega y amigo Theodore Gill, cuando en Cuba aun no existían entidades de ese tipo donde pudieran ser depositados. Algunos ejemplares que el mismo naturalista conservó hoy no tienen paradero conocido.

Los estudios de la biodiversidad requieren de consultas obligadas para asegurar que las especies sean correctamente identificadas. Los conservadores que trabajan con colecciones de historia natural tienen en sus manos la responsabilidad, no solo de mantenerlas en buen estado de conservación para su consulta cotidiana por parte de la comunidad científica especializada, sino también de propiciar que el conocimiento que guardan llegue de forma factible y práctica a los estudiosos. Por esto pensamos que se hace necesario crear catálogos digitales y otros productos informáticos que pongan a disposición libre la información sobre las colecciones y en ello hemos trabajado desde hace unos años (Ibarzábal et al., 2010; Orozco et al., en edición).

\section{OBJETIVO}

- Difundir información recopilada sobre los tiburones tipo cubanos por medio de un catálogo ilustrado.

\section{MATERIALES Y MÉTODOS}

La información utilizada para confeccionar el catálogo ilustrado ha sido organizada partiendo del Proyecto "Multimedia Tipos Marinos Cubanos" (Orozco et al., en edición), una amplia recopilación digital sobre los especímenes tipo de las especies marinas cubanas pertenecientes a diferentes táxones. Para su publicación, se utilizaron los datos de la literatura original donde se encuentran las descripciones y las figuras usadas en las mismas.

En cada una de las especies incluidas se ofrecen los datos del holotipo y paratipos partiendo de la literatura original ya mencionada, de la cual también se tomaron las ilustraciones, las que fueron redimensionadas al tamaño 800X600 utilizando Adobe Photoshop CS5. Se ofrece el ordenamiento taxonómico y los siguientes datos: autor, año de la descripción, información bibliográfica completa, sinonimias (en caso de haberlas), institución depositaria de los tipos con sus números catalográficos, localidad tipo, fecha de colecta, profundidad a que se encontraron y la expedición en que se realizó la colecta.; en algunos casos también se registra el nombre del colector. Cuando faltan algunos de los datos enunciados es porque no se encuentran en dicha referencia.

\section{RESULTADOS}

Catálogo ilustrado de los tipos de tiburones cubanos.

Clase CHONDRICHTHYES Huxley, 1880

Subclase HOLOCEPHALI (Bonaparte, 1832-41)

Orden CHIMAERIFORMES

Familia CHIMAERIDAE Bonaparte, 1831 
Chimaera cubana Howell Rivero, 1936. Figura 1.

Holotipo: MCZ 1464/34037. Paratipo: MCZ 1385.

Institución Depositaria: Museo de Zoología Comparada, Universidad de Harvard, EE. UU.

Localidad: Bahía de Matanzas, Cuba.

Título: Some new, rare and litlle known fishes from Cuba.

Referencia: Proc. Boston Soc. Nat. Hist. vol. 41: 61.

Subclase ELASMOBRANCHII Bonaparte, 1838

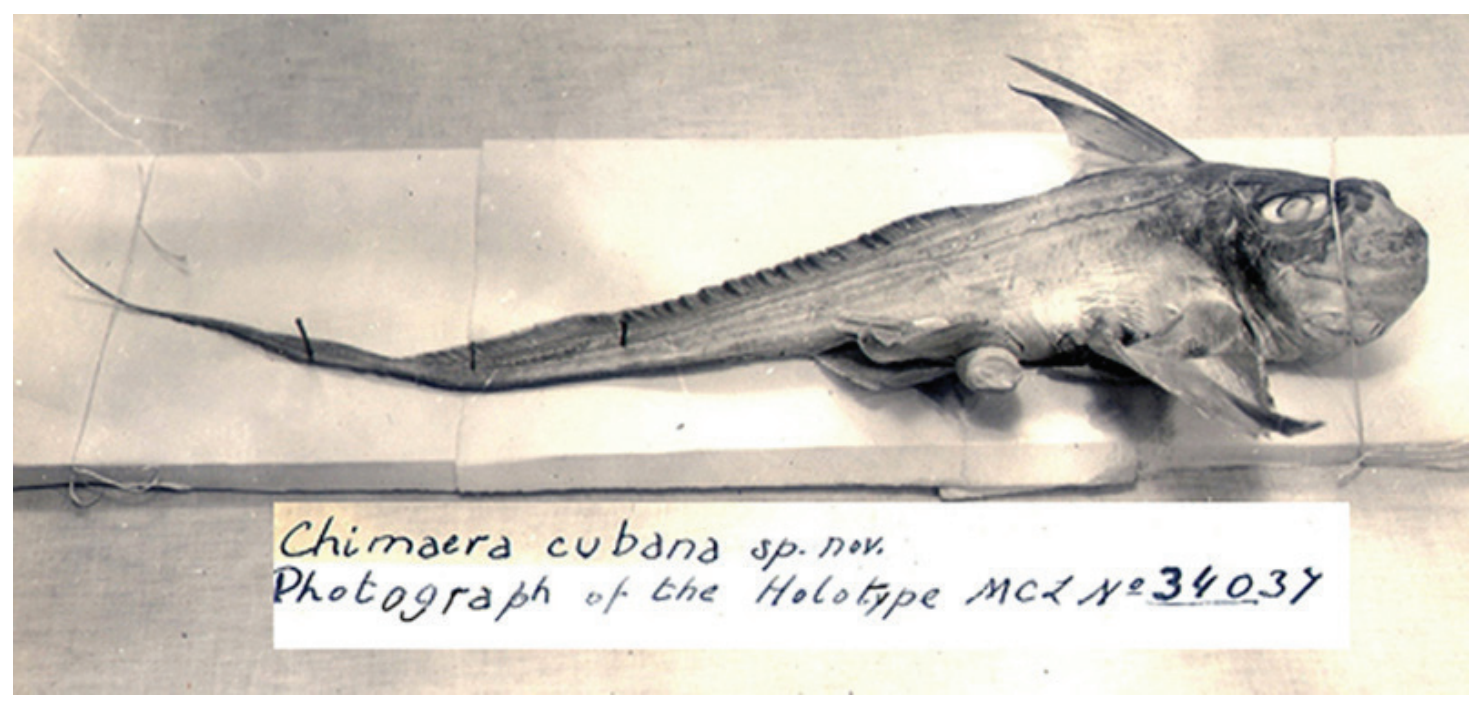

Figura 1. Foto del espécimen tipo de Chimaera cubana, tomada por Howell Rivero, autor de la descripción de la especie, con anotaciones de su puño y letra. La fotografía fue donada por la familia del Dr. Howell Rivero al Acuario Nacional de Cuba entre otros materiales originales de su trabajo como ictiólogo.

Orden SQUALIFORMES Compagno, 1973

Familia SQUALIDAE de Brainville, 1816

Squalus cubensis Howell-Rivero, 1936. Figura 2.

Holotipo: MCZ 1458. Paratipos: MCZ 1459-1462.

Institución depositaria: Museo de Zoología Comparada, Universidad de Harvard, EE. UU.

Localidad tipo: Estrecho de la Florida y Cuba.

Profundidad: 325 brazas.

Título del trabajo: Some new and litlle-known fishes from Cuba.

Referencia: Proc. Boston Soc. Nat. Hist., vol. 41(4): 45. 


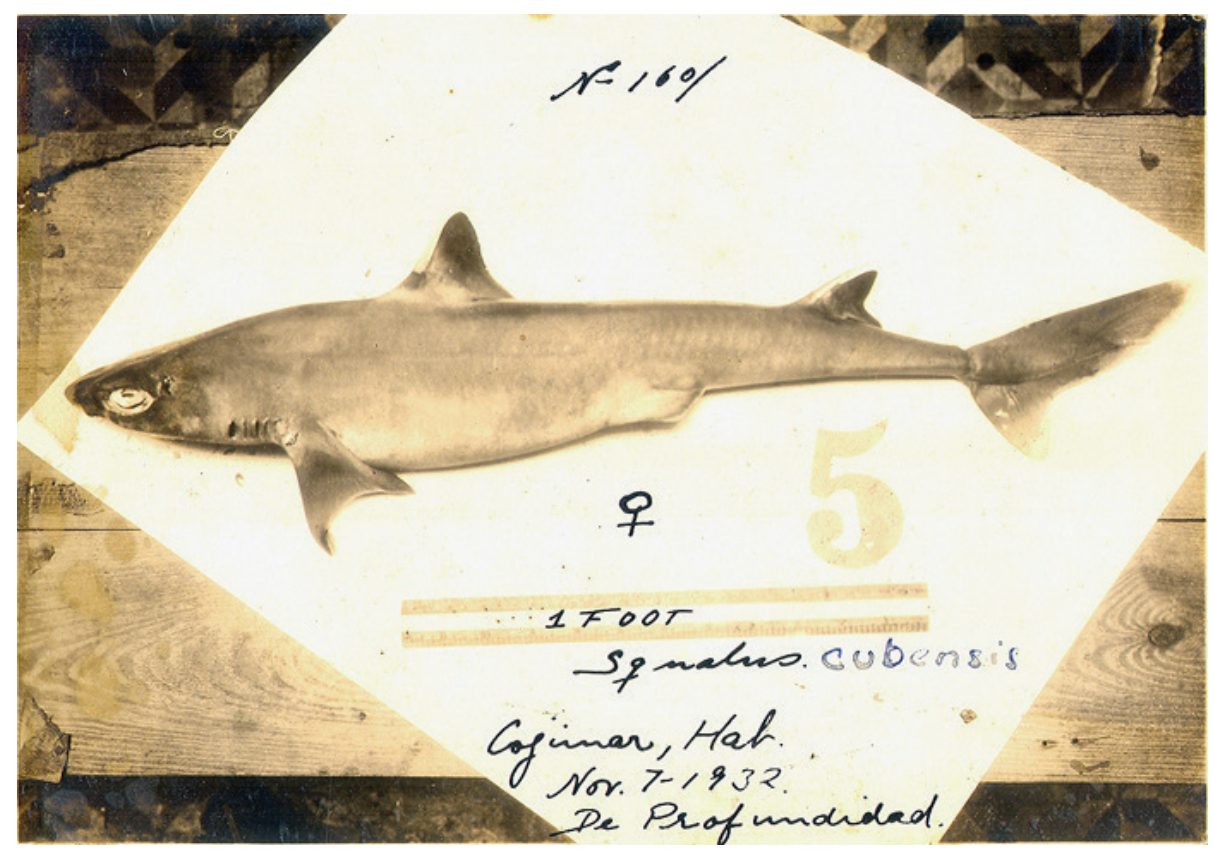

Figura 2. Foto del espécimen tipo de Squalus cubensis, tomada por Howell Rivero, autor de la descripción de la especie, con anotaciones de su puño y letra. La fotografía fue donada por la familia del Dr. Howell Rivero al Acuario Nacional de Cuba.

Etmopterus hillianus (Poey, 1861)

Sinonimia: Spinax hillianus Poey, 1861.

Ejemplar de Poey No. 617. Holotipo: MCZ 1025 (hembra).

Institución Depositaria: Museo de Zoología Comparada, Universidad de Harvard, EE. UU.

Localidad: Cuba.

Título del Trabajo: Cap. XLIX. "Espéces Nouvelles (1)" "Poissons de Cuba".

Referencia: Memorias de la Historia Natural de la Isla de Cuba, v.2: 340-342.

\author{
Orden CARCHARHINIFORMES
}

Familia SCYLIORHINIDAE Gill, 1862

Apristurus riveri Bigelow y Schroeder, 1944

Holotipo: MCZ 36092.

Institución Depositaria: Museo de Zoología Comparada, Universidad de Harvard, EE. UU. 
Localidad: frente a Cruz del Padre, Villa Clara, Costa N de Cuba.

Profundidad: 580 brazas.

Título: New sharks from the western North Atlantic.

Referencia: Proc. New England Zool. Club vol. 23: 23.

Scyliorhinus torrei Howell Rivero, 1936. Figura 3.

Holotipo: MCZ 1457-S

Institución Depositaria: Museo de Zoología Comparada, Universidad de Harvard, EE. UU.

Localidad: frente a La Habana, Cuba.

Título: Some new, rare and litlle known fishes from Cuba.

Referencia: Proc. Boston Soc. Nat. Hist. vol. 41: 43.

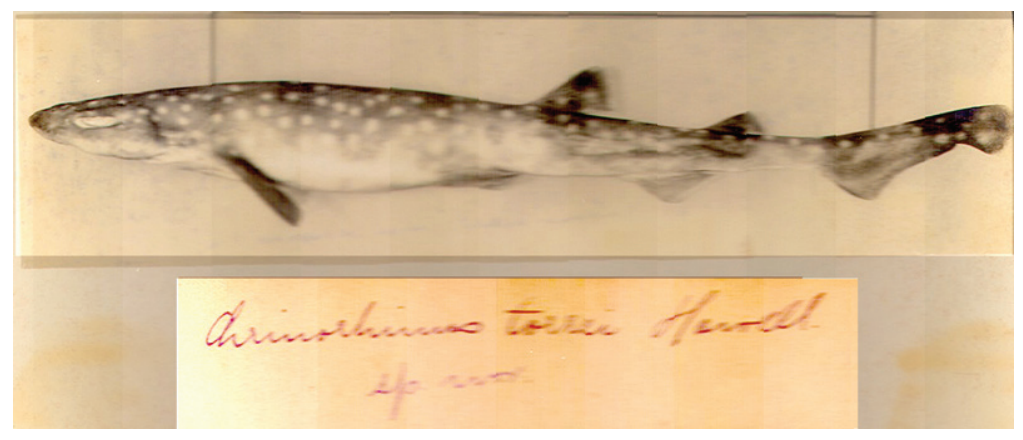

Figura 3. Foto del espécimen tipo de Scyliorhinus torrei tomada por Howell Rivero, autor de la descripción de la especie, con anotaciones de su puño y letra. La fotografía fue donada por la familia del Dr. Howell Rivero al Acuario Nacional de Cuba.

Familia TRIAKIDAE Gray, 1851

Allomycter dissutus Guitart Manday, 1972

Sinonimia: Mustelus canis insularis Heemstra, 1997.

Ejemplar único (perdido).

Localidad: Bahía de Matanzas, Cuba.

Profundidad: $366 \mathrm{~m}$.

Título: Un nuevo Género y Especie de tiburón de la Familia Triakidae.

Referencia: Poeyana 99: 1-4. 
Familia PROSCYLLIIDAE Fowler, 1941

Eridacnis barbouri (Bigelow y Schroeder, 1944)

Sinonimia Triakis barbouri Bigelow y Schroeder, 1944.

Holotipo: MCZ 36099. Paratipos: MCZ 36134, 36135, 36137-36141, 36143-36151, 36214 y 63201.

Institución Depositaria: Museo de Zoología Comparada, Universidad de Harvard, EE. UU.

Localidad: frente a Ciego de Ávila y Villa Clara, costa N de Cuba.

Título: New sharks from the western North Atlantic.

Referencia: Proc. New England Zool. Club vol. 23: 27.

Familia CARCHARHINIDAE Jordan y Evermann, 1896

Carcharhinus acronotus (Poey, 1860)

Sinonimia: Squalus acronotus Poey.

Ejemplar de Poey No. 555. Holotipo único (perdido).

Institución Depositaria: Desconocida.

Localidad: Cuba.

Título: Cap. XLIX. "Espéces Nouvelles (1)" "Poissons de Cuba".

Referencia: Memorias de la Historia Natural de la Isla de Cuba, v.2: 335-336 (pl. 18, f. 3, 4).

Carcharhinus longimanus (Poey, 1861)

Sinonimia: Squalus longimanus Poey.

Ejemplar de Poey No. 123. Holotipo único (perdido).

Institución Depositaria: Desconocida.

Localidad: Cuba.

Título: Cap. XLIX. "Espéces Nouvelles (1)" "Poissons de Cuba".

Referencia: Memorias de la Historia Natural de la Isla de Cuba, v.2: 338-339.

Carcharhinus perezi (Poey, 1876)

Sinonimia: Platypodon perezii Poey.

Ejemplar de Poey No. 570. Sintipos: 6 (perdidos).

Institución Depositaria: desconocida. 
Localidad: Cuba.

Título: ENUMERATIO PISCIUM CUBENSIÜM.Parte Tercera.

Referencia: Anales de la Sociedad Española de Historia Natural, Madrid, vol.5: 390 (194)-393 (197) (LÁMINA XIV, FIGURAS 2 y 3).

Carcharhinus signatus (Poey, 1868)

Sinonímia Hypoprion signathus, Poey, 1868

Ejemplar de Poey No. 567. Mandíbulas solamente (perdidas).

Institución Depositaria: desconocida.

Localidad: Cuba.

Título: Sinopsis Piscium Cubensium (ó Catalogo de los Peces de la Isla de Cuba).

Referencia: Repertorio Físico-Natural de la Isla de Cuba, vol. 2: 452 (Plate 4, Figs. 7-8).

Negaprion brevirostris (Poey, 1868)

Sinonimia Hypoprion brevirostris, Poey.

Ejemplar de Poey No. 554 (perdido).

Institución Depositaria: desconocida.

Localidad: Cuba.

Título: Sinopsis Piscium Cubensium (ó Catálogo de los Peces de la Isla de Cuba).

Referencia: Repertorio Físico-Natural de la Isla de Cuba, vol. 2: 451-452 (Tabla 4, Figs. 5, $6,20)$.

Rhizoprionodon porosus Poey, 1861

Sinonimia Squallus porosus Poey.

Ejemplar de Poey No. 410 (perdido).

Institución Depositaria: desconocida.

Localidad: Cuba.

Título: Cap. XLIX. "Espéces Nouvelles (1)" "Poissons de Cuba".

Referencia: Memorias de la Historia Natural de la Isla de Cuba, v.2 : 339-340 (P1.19,

Figs. 11-12).

Orden RAJIFORMES L. S. Berg, 1940

Familia RAJIDAE de Blainville, 1816 
Holotipo: MCZ 36374 (333 mm TL). Hembra.

Institución Depositaria: Museo de Zoología Comparada, Universidad de Harvard, EE. UU.

Localidad: Bahía de Matanzas, Lat: 2312’ 0” N; Long: 81²3’ 0” W.

Profundidad: 285 brazas.

Expedición: Harvard-Havana Atlantis Expedition.

Fecha: 11-5-1939.

Título: No.7.- New genera and species of batoid fishes.

Referencia: Sears Found Jour. of Mar. Res. 7(3): 559 Figs 2-6.

Cruriraja atlantis Bigelow y Schroeder, 1948

Holotipo: MCZ 36320 (336 mm TL). Hembra.

Institución Depositaria: Museo de Zoología Comparada, Universidad de Harvard, EE. UU.

Localidad: frente a Matanzas, Lat: $23^{\circ} 11$ `30” N; Long: 81²3`01” W.

Prof.: 375 brazas.

Expedición: Harvard-Havana Atlantis Expedition.

Fecha: 9-5-1939.

Título: No.7.- New genera and species of batoid fishes.

Sears Found Jour. of Mar. Res. 7(3): 550 Figura 3.

Cruriraja poeyi Bigelow y Schroeder, 1948

Holotipo: MCZ 36324 (330 mm TL). Hembra con la cola partida.

Institución Depositaria: Museo de Zoología Comparada, Universidad de Harvard, EE. UU. Localidad: Frente a Punta Alegre, Ciego de Avila, Lat: $22^{\circ} 48^{`} 01^{\prime}$ N Long: $78^{\circ} 50$ 01” W

Prof.: 210 brazas

Expedición: Harvard-Havana Atlantis Expedition.

Fecha: 11-3-1938.

Título: No.7.- New genera and species of batoid fishes.

Sears Found Jour. of Mar. Res. 7(3): 555 Figs 1-5.

Fenestraja atripina (Bigelow y Schroeder, 1950)

Sinonimia Breviraja atripina Bigelow y Schroeder, 1950.

Holotipo: MCZ 36370. Paratipos: MCZ (2).

Institución Depositaria: Museo de Zoología Comparada, Universidad de Harvard, EE. UU. Localidad: Atlantis Station 3443, frente a Villa Clara, Lat. $23^{\circ} 22^{`}$ N., Long. $79^{\circ} 53^{`} \mathrm{~W}$ 
Prof.: 325 brazas.

Expedición: Harvard-Havana Atlantis Expedition.

Título: No.7.- No.7.- New and little known cartilaginous fishes from the Atlantic.

Bull. Mus. Comp. Zool., vol. 103: 390-393 (Plate 3).

Fenestraja cubensis (Bigelow y Schroeder, 1950)

Sinonimia Breviraja cubensis Bigelow y Schroeder, 1950.

Holotipo: MCZ 36443 (macho, 210 mm de longitud).

Institución Depositaria: Museo de Zoología Comparada, Universidad de Harvard, EE. UU.

Localidad: Atlantis Station 3451, frente a Villa Clara, Lat. $23^{\circ} 20^{`}$ N., Long. $79^{\circ} 59^{`} \mathrm{~W}$

Expedición: Harvard-Havana Atlantis Expedition.

Título: No.7.- New and little known cartilaginous fishes from the Atlantic.

Bull. Mus. Comp. Zool., vol.103: 394-396 (Plate 4).

\section{LITERATURA CITADA}

Bigelow, H. B. y W. C. Schroeder. 1944. New sharks from the western North Atlantic. Proc.New England Zool.Club, vol. 23: 21-36.

Bigelow, H. B. y W. C. Schroeder. 1948. New genera and species of bathoid fishes. Sears Found Jour. Mar. Res., 7(3): 550, 555 y 559.

Bigelow, H. B. y W. C. Schroeder. 1950. No.7.- New and little known cartilaginous fishes from the Atlantic. Bull. Mus. Comp. Zool., vol. 103: 390-396.

Chesnut, D. 2014. The Sloans Valley Member (Paragon Formation) of Kentucky: Vertebrata. Disponible desde: http://www.uky.edu/OtherOrgs/KPS/stratigraphicfauna/ sloansvalleymacrofauna/pages/sloansvalleyvertebrata.html.

Guitart Manday, D. J. 1966. Nuevo nombre para una Especie de tiburón del Género Isurus (Elasmobranchii: Isuridae) de aguas cubanas. Poeyana, 15: 1-9.

Guitart Manday, D. J. 1972. Un nuevo Género y Especie de tiburón de la Familia Triakidae. Poeyana, 99: 1-4.

Howell Rivero, L. 1936. Some new and little-known fishes from Cuba. Proc. Boston Soc. Nat. Hist, vol. 41(4): 43-68.

Ibarzábal Bombalier, D., M.V. Orozco, E. Gutiérrez, R. Cortés, Y. Alfonso, N. Barbán, S. A. Gallardo, C. J. Alonso y A. Blanco. 2010. Multimedia "Corales Pétreos de Cuba". Acuario Nacional de Cuba, Agencia de Medio Ambiente, CITMA. ISBN 978-959-300-007-9.

Müller, J. y F. G. J. Henle.1838-41. Systematische Beschreibung der Plagiostomen. Berlin. i-xxii+1-200, 60 pls.(Pp. 1-28 published in 1938, reset pp. 27-28, 29-102 in 1939, i-xxii+ 103-200 in 1841). 
Nelson, J. S. 1994. Fishes of the World, Third Edition. xvii + 600. Disponible desde: http://www. itis.gov/servlet/SingleRpt/RefRpt?search_type=publication\&search_id=pub_id\&search id_value $=1486$.

Orozco, M.V., E. Gutiérrez, R. Cortés, Y. Alfonso y A. Blanco. (en edición): Multimedia “Tipos Marinos Cubanos". Acuario Nacional de Cuba, Agencia de Medio Ambiente, CITMA.

Poey Aloy, F. 1860-61. Cap. XLIX. "Espéces Nouvelles (1)" "Poissons de Cuba". Memorias de la Historia Natural de la Isla de Cuba, vol. 2: 335-342.

Poey Aloy, F. 1868. Sinopsis Piscium Cubensium (o Catálogo de los Peces de la Isla de Cuba). Repertorio Físico-Natural de la Isla de Cuba, vol. 2: 451-452.

Poey Aloy, F. 1876. Enumeratio Piscium Cubensiüm. Parte Tercera. Anales de la Sociedad Española de Historia Natural, Madrid, vol. 5: 390 (194)-393 (197).

Van Der Laan, R., W. N. Eschmeyer y R. Fricke. 2014. Family-group names of recent fishes. Disponible desde: http://biotaxa.org/Zootaxa/article/view/zootaxa.3882.1.1/10480.

[Recibido: 10 de enero, 2015. Aceptado para publicación: 18 de mayo, 2015] 